\title{
El psicólogo y la procreación aplazada: Consideraciones acerca de la criopreservación y de una calidad óptima de gametos
}

\author{
Daniel M. Campagne \\ Universidad Nacional de Educación a Distancia, Madrid, España
}

\begin{abstract}
Resumen: La procreación aplazada y la criopreservación de óvulos y esperma son tendencia en auge. Aunque es legítima la "congelación social" (no por razones médicas) de gametos, se debaten aspectos éticos y sociales. Las técnicas de reproducción asistida (TRA) y la criopreservación, combinadas con la matriz sustituta, se acercan a cierta ectogénesis. Los aspectos psicológicos y morales que surgen antes, durante y después de este proceso implican al psicólogo clínico quien debe estar informado sobre lo que las criotécnicas pueden y podrán hacer. Comentamos las responsabilidades hacia el niño-por-nacer, tanto el no nacido como el aún no concebido. Estos desarrollos rápidos en la reproducción humana hacen aparecer indicaciones médicas, sociales y legales de una separación formalizada entre la procreación y la actividad sexual, diferenciación que irá especificando las responsabilidades en la primera mientras respete la elección personal en la segunda. Son de interés social las implicaciones psicológicas de la reproducción aplazada.
\end{abstract}

Palabras clave: Calidad de gametos ; criopreservación; ética; madre sustituta; niño no concebido; procreación aplazada

The psychologist and delayed reproduction: Reflections on cryopreservation and optimal gamete quality

\begin{abstract}
Delaying reproduction and cryopreserving oocytes and sperm is a tendency on the rise. Although "social freezing" (not for medical reasons) of gametes is legitimate, its ethical and social aspects are being debated. Assisted reproduction and cryopreservation, in combination with gestational surrogacy, are nearing ectogenesis. Psychological and moral questions coming up before, during and after this process implicate the clinical psychologist who must keep up with what cryotechniques are, or will be, capable of. We discuss the responsibilities of delayed parenting towards the child-to-be, not only the not-yet-born but also the not-yet-conceived. These rapid developments in reproduction are producing medical, social and legal indications of a formal separation between procreation and sexual activity, a differentiation which will specify responsibilities in the first whilst respecting personal choice in the second. The individual reasons for and psychological consequences of delayed reproduction are of social interest.
\end{abstract}

Keywords: Cryopreservation; delayed parenting; ethics; future child; gamete quality; surrogacy.

\section{Introducción}

El aplazamiento en tener hijos es una evolución de nuestros tiempos, provocada por diversos tipos de desarrollos personales, sociales y legales que cada vez más necesitan de atención sanitaria y económica. En los países desarrollados, la edad media del primer embarazo se

Recibido: 15 octubre 2014; aceptado: 1 diciembre 2014.

Correspondence: Daniel M. Campagne, Universidad Nacional de Educación a Distancia, Facultad de Psicología, Juan del Rosal 10, 28040 Madrid, España. Correo-e: danplaton@hotmail.com. Tel.: +34965873120 . aproxima a los 30 años, y el número de mujeres mayores de 35 años que intentan concebir un primer hijo está creciendo rápidamente. Las consecuencias de una crianza en edad más avanzada ya reclaman la atención de gobiernos y profesionales y están fomentando una discusión multi-disciplinaria sobre las cuestiones éticas relacionadas, entre ellas una fundamental:" $i S e$ puede recomendar la congelación de esperma y óvulos en más casos, o tal vez en general?"

Además de revisar los datos que arroja la investigación en cuanto a posibles respuestas a estas preguntas, procedemos a una breve revisión del estado actual de los procedimientos y técnicas existentes e inminentes, que 
puede ayudar a clarificar en qué medida ellos reflejen una realidad actual que se puede considerar aplicable a la población en general, o si se trata de posibilidades teóricas aun sin las suficientes garantías. Tanto las posibilidades reales como las futuras afectan a las personas confrontadas con la procreación aplazada y eleva la probabilidad de alteraciones psicológicas y, por ende, la intervención del psicólogo clínico.

Con la legalización del control de natalidad, nuestra sociedad empezó con esta separación formal entre la procreación y la actividad sexual, pero la reproducción en sí aún hoy depende mayoritariamente del azar o de la intervención ad hoc. Sin embargo, la tecnología disponible alienta a todos los interesados a contemplar si no se debe dar más importancia a posibles medidas preventivas en nuestra búsqueda de soluciones para los riesgos del aplazamiento en la concepción y sus consecuencias para los hijos. Se acude cada vez más a la criopreservación de oocitos y esperma "preventiva" o "defensiva", llamada "congelación social", en contraste con la "congelación médica", lo cual demuestra por un lado la existencia de un problema social a causa del choque de la paternidad/maternidad con las exigencias de nuestra sociedad y, por otro, de una concienciación en aumento en cuanto a las consecuencias de una procreación a edad más avanzada. Desde 2004, las clínicas de fertilidad se están adaptando a esta realidad con servicios que pretenden ayudar a preservar la fertilidad en el tiempo (Gootman, 2012). Aunque aún podría considerarse futurista la noción de ectogénesis, entendida como producir niños en laboratorios, en realidad se está muy cerca - si no ya - de poder hacerlo, con procedimientos y técnicas que se emplean a gran escala y se afinan continuamente desde hace décadas. Libros y artículos en la prensa popular predicen un futuro de bebés clonados, genéticamente "ajustados" y producidos en laboratorios. Parte de ello es ya técnicamente posible y en algunos casos viable. Sin embargo, desde un punto de vista médico, legal y económico, en la actualidad es mucho más simple usar un útero sustituto en vez de un útero de laboratorio. Aprovechándose de la disposición de mujeres pobres a rentabilizar su útero con su alquiler a terceros, países como la India han visto crecer una "industria" alrededor de la sustitución de útero. La ética y el control de calidad aquí son aspectos por resolver. Esta tendencia apunta a que se está aceptando un tipo biológico de la ectogénesis, un desarrollo del que sus consideraciones morales están por desarrollar.

Los servicios técnicos ya disponibles dan un realismo cada vez más sólido a la maternidad o paternidad aplazada. Se vislumbra que, a una edad joven, mujeres y hombres que están ocupados con su carrera podrían sin problema congelar óvulos y esperma, seguir con su carrera $y$, en cuanto decidan, concebir un niño con TRA y encargar el embarazo a una madre gestacional.

Para la mayoría de las personas, cualquier cuestión que afectara a la fertilidad no suele ser fácil. Las cuestiones o problemas de infertilidad pueden afectar seriamente la salud psicológica de una persona, independientemente de las consecuencias económicas (Campagne, 2006). Psicológicamente, una persona considera su fertilidad indudable hasta que se enfrenta con lo contrario. Una persona que se ve presentada con problemas de infertilidad sólo raras veces logra evitar un estrés sustancial y dudas de sí mismo. Incluso con la ayuda de los avances considerables que se han conseguido en los pasados tres decenios en cuanto al tratamiento de la infertilidad en general, con técnicas de fertilización in vitro (FIV) y la inyección intracitoplasmática de espermatozoides (ICSI) en particular, la confrontación personal con la infertilidad no se ha vuelto mucho más fácil de llevar, y tampoco se han incrementado las tasas de éxito de las TRA tanto que se pueda ya garantizar el nacimiento de un niño sano.

Las técnicas que se desarrollaron para solventar problemas médicos de infertilidad se pueden aplicar a problemas sociales de fertilidad, entre los que se consideran la procreación aplazada por decisión propia. En la actualidad, entre las soluciones tanto para la infertilidad médica como la procreación aplazada social, se incluyen cada vez más la criopreservación de espermatozoides, oocitos, embriones o tejido reproductivo. La congelación ha resultado de gran ayuda aunque no una panacea. Los investigadores en criobiología han analizado muchos aspectos de la criopreservación y sus consecuencias, los cuales aún producen cautela y necesitan más investigación y ajustes en la práctica clínica. Sin embargo, una colección prometedora de posibilidades abre la puerta a una más amplia escala de aplicación de la criopreservación como una técnica de uso general que se podría aplicar para frenar o minimizar el efecto negativo producido por el tiempo sobre los gametos.

\section{Causas y consecuencias psicológicas de la procreación aplazada}

El hecho de decidir conscientemente que uno quiere aplazar tener un primer hijo es un asunto psicológico. En muchos casos la decisión es inconsciente, y sólo se manifiesta en el aplazamiento. La consideración consciente o subconsciente de sus ventajas es un proceso psicológico, aunque fuertemente influenciado por factores sociales y económicos (Daly y Bewley, 2013). Aunque la procrea- 
ción aplazada, la TRA, y la criopreservación son aceptadas socialmente, presentan muchos factores psicológicos y emociones conflictivas, amén de las consecuencias físicas (Sheiner, Shoham-Vardi, Hershkovitz, Katz, y Mazor, et al., 2001). Más de la mitad de las mujeres que se someten a tratamientos de infertilidad experimentan problemas de índole emocional, fisiológico, psicológico y social (Schardt, 2005). Tanto mujeres como hombres tienen que hacer frente a las influencias de la familia y el entorno social, a su propia reacción al diagnóstico y la propuesta de tratamiento, a la influencia de la religión en cuanto al tratamiento, la valoración de su vida sexual, y a su sentido de autovaloración y auto-aceptación. Es posible que el sector de la población más propenso a aplazar la procreación se caracterice por presentar un "constructo con propiedades transdiagnósticas" (Sandin, Chorot y Valiente, 2012) de relevancia psicopatológica.

Como decimos antes, para muchas personas un problema o una duda relacionados con la fertilidad no son de fácil solución. Un diagnóstico de infertilidad a menudo provoca una reacción de estrés agudo, seguida por la tensión y el agotamiento nervioso que se desarrollan con el tratamiento de infertilidad. Por ende, se combinan respuestas fisiológicas y psicológicas, tanto en hombres como en mujeres (Chachamovich, Chachamovich, Fleck, Cordova, y Knauth, et al., 2009). Las TRA aumentan las posibilidades de tener un embarazo múltiple, con sus riesgos asociados y más estrés, factores físicos y decisiones difíciles (Heffner, 2004). En el caso de que se presenten problemas dentro del embarazo, se desarrollan emociones como sentimientos de culpa, ira, tristeza, resentimiento, impotencia y miedo, en una proporción más elevada que en embarazos espontáneos.

Contrario a las creencias habidas en cuanto a la supuestamente más baja vulnerabilidad psicológica de los hombres en cuanto a la infertilidad involuntaria, un estudio de sección transversal sueco de 2010 encontró que tanto hombres como mujeres en el grupo de TRA infructuosa, no se distinguían en ninguno de los parámetros medidos de calidad de vida, ambos grupos mostrando más ansiedad, depresión, menor bienestar psicológico general y un menor sentido de coherencia que el grupo exitoso o de control (Johansson, Adolfsson, Berg, Francis, y Hogström et al., 2010). Esto significa que los padres no se libran de las connotaciones psicológicas negativas de la infertilidad. Un estudio danés de 2013 ( $N=$ 1.406) informa de síntomas de depresión mayor en el $11,6 \%$ de las mujeres y el $4,3 \%$ de los hombres, asociadas de forma significativa con un incremento del estrés relacionado con la infertilidad, a niveles individuales y de pareja (Peterson, Sejbaek, Pirritano, y Schmidt, 2014).
¿En qué momento de la vida edad, enfermedad, accidentes, estilo de vida y el entorno empiezan a dañar esperma y oocitos? Una edad avanzada paterna ya es asunto de preocupación para organizaciones nacionales de salud, a cuenta de varios riesgos incrementados. Los efectos de una edad avanzada materna están documentados desde hace mucho tiempo, pero la investigación sigue descubriendo más áreas donde ejerce un efecto negativo.

A pesar de datos contundentes, no se ha prestado aún mucha atención a la pregunta de si la criopreservación de gametos ya puede o debe ser propuesta como remedio para el envejecimiento reproductivo en una mayoría de casos. En muchos países, las asociaciones profesionales sólo recientemente empiezan a reconocer las razones legitimadas para la congelación social de óvulos. En 2012, la European Society of Human Reproduction and Embryology (ESHRE) se pronunció a favor de la criopreservación preventiva (ESHRE, 2012). Pero una cuestión fundamental parece digna de un debate más profundo, aunque no todas las implicaciones técnicas se hayan solucionado en la actualidad. ¿Por qué permitiría un hombre o una mujer que su fertilidad se reduzca o baje de calidad si es posible recolectar sus gametos de mejor calidad en su mejor momento y guardarlos para un uso posterior? Si las personas necesitan primero abrirse camino en su carrera o negocio, o si son obligados a vivir en un entorno contaminado o trabajan con sustancias peligrosas, si fuman o consumen drogas, sufren accidentes o enfermedad, parece no sólo sentido común sino una responsabilidad moral que intenten proteger sus futuros hijos de estas circunstancias vitales.

\section{Las consecuencias de la procreación aplazada para la calidad de los gametos}

En fertilidad, la edad tiene un significado diferente. Aunque a todos los demás efectos una mujer de 35 años es joven, en cuanto a fertilidad es de Edad Materna Avanzada (EMA). Un hombre de 40 estará en la flor de la vida, pero para la fertilidad es de Edad Paterna Avanzada (EPA).

Una mujer nace con todos sus óvulos mientras un hombre produce esperma durante una parte significativa de su vida. La investigación reciente sugiere que las mujeres puede que tengan células madre que puedan convertirse en nuevos óvulos durante su vida, pero los mecanismos aún no están claros. Así que las consecuencias de aplazar la reproducción difieren para mujeres y hombres, teniendo más efecto sobre las primeras. Además, difieren también las posibles consecuencias negativas para el niño, dependiendo de las características de los 
gametos de cada progenitor. Alrededor de los 35 años de edad, las mujeres experimentan una bajada de fertilidad relativamente fuerte. Más importante y tal vez no tan conocido es, que el óvulo más viejo no tiene los mismos contenidos originales que el más joven. Como indicamos ya, es un error común pensar que la TRA remedie o altere de forma alguna este patrón de modificación química e impronta parental que ocurre en el proceso epigenético, eso es, desde la edad más temprana de la mujer. Los óvulos que son inducidos a madurar y/o forzados a fertilización a una edad de 30, 35 o 40 años, no aportan un genoma fresco al niño futuro. Los cambios acumulados afectan a la regulación de la expresión de genes de impronta durante el desarrollo embriónico, lo cual resulta en un incremento de una predisposición a enfermedades raras. Hasta la fecha, la única forma de remediar el envejecimiento de los óvulos es la donación.

Una vez que la mujer descubra que tiene problemas de infertilidad, su reacción depende de la edad. Las de menos de 35 años se veían motivadas a intentar mejorar su fertilidad con el abandono de comportamientos que la reducen (p.ej. fumar, aplazar buscar atención médica en cuanto experimentan retrasos en la concepción) mientras la mujer más mayor no mostraba esta inclinación (Fulford, Bunting, Tsibulsky, y Boivin, 2013)

Los hombres tienen una reducción de fertilidad parecida pero más gradual. Un estudio de 2013 juntó 2.276 ciclos de inseminación intrauterina según la edad del hombre. Se encontró una tasa de embarazo más bajo para el grupo de hombres de más de 50 años. Se estableció una correlación negativa entre el incremento de edad y el volumen total de células motiles. La morfología del esperma fue decisiva a partir de los 45 años. El estilo y las condiciones de vida demostraron ser de una importancia relevante para la calidad de gametos masculinos que se encuentra especialmente afectada en hombres mayores. Un estudio noruego de $2013(N=14.832)$ analizó las características relevantes de padres primerizos de edad avanzada (35-39 años) y edad muy avanzada (40 y más años), en comparación con hombre de 25-34 años. De los futuros padres más mayores en el grupo de estudio había más solteros o que no convivían, con una actitud saludable negativa (exceso de peso, obesidad, fumador, toma habitual de alcohol), con problemas de salud física y mental (lumbalgia, enfermedades cardiovasculares, hipertensión, problemas de sueño, síntomas depresivos anteriores), con pocos contactos sociales y poca satisfacción con la relación de pareja. Los autores también encontraron que, en general, los hombres mayores tenían su primerizo con una mujer mayor (Nilsen, Waldenström, Rasmussen, Hjelmstedt, y Schytt, 2013). Por lo tanto, resulta que no sólo el esperma de hombres mayo- res se ve afectado en mayor medida por los efectos negativos de la edad misma, sino que en este grupo se encuentran sobrerrepresentados los estilos de vida poco sanos con efectos negativos sobre el esperma.

\section{Las consecuencias de la procreación aplazada para el niño}

El desarrollo y el futuro del niño dependen de la calidad de los gametos con los que ha sido concebido. Un aplazamiento, especialmente si es significativo, puede comprometer esta calidad. La edad ovárica biológica depende de varios factores de carácter médico, estilo de vida, genético, autoinmune e idiopático. La heredabilidad es importante (Younis, 2012). El envejecimiento ovárico medible empieza sobre los 30 años, para ser marcadamente más pronunciado sobre los 35 y alcanzar niveles casi infértiles después de los 40 (Liu y Case, 2011; Kimberly, Case, Cheung, Sierra, AlAsiri, et al., 2012). Una mujer debería tener esto presente y también el hecho de que, según avance la edad maternal, también lo hace la posibilidad de que los hijos se verán afectados por una anormalidad cromosómica, como una trisomía. De un riesgo estadístico para la trisomía 21 (Síndrome de Down) de 1/1352 para una edad maternal de 25 años, subió a 1/97 para la edad de 40 años (Snijders, Sundberg, Holzgreve, Henry, y Nicolaides, 1999). Un estudio danés de 2013 encontró que el propio tratamiento de fertilidad producía un incremento pequeño en anormalidades cromosómicas, tanto de novo como genéticas. (Bay, Mortensen, Hvidtjørn, y Kesmodel, 2013). Existen otros riesgos para la salud y el futuro del feto nacido de una madre EMA, entre ellos el aborto espontáneo, nacimiento prematuro, ser gemelo o trillizo, parálisis cerebral, y peso bajo al nacer con sus probables problemas de salud más tardíos (Reynolds, 2013).

Como ya indicado, hombres mayores tienen más daños en el ADN del esperma, asociados con defectos de desarrollo y enfermedades genéticas en su descendencia . Se vio que el volumen y la motilidad del esperma se reducen de forma continuada entre los 22 y 80 años, sin señal de umbral alguno y se manifiesta especialmente desde los 40 años (Silva, Oliveira, Petersen, Mauri, Massaro, et al., 2012). Una avanzada edad paterna se relaciona con un incremento del riesgo de aborto espontaneo y un riesgo más alto de condiciones dominantes autosomales, trastornos del espectro autista, y esquizofrenia (Liu y Case, 2011). La razón está en el hecho que padres pasan mucho más mutaciones genéticas nuevas que madres, con el número de nuevas mutaciones que se transmiten incrementándose exponencialmente con la edad paterna. Uno de 36 años transmite el doble de mutacio- 
nes a su hijo que un hombre de 20, y uno de 70 años ocho veces más (Kong, Frigge, Masson, Besenbacher, Sulem, et al., 2012). El por qué hombres mayores incorporan muchas más mutaciones (errores) en su esperma que hombres jóvenes es el resultado de la forma en que se produce el esperma. Los espermatozoos son el resultado de la división de células gonadales. Cada división puede producir un error de copia ADN en este proceso permanente de reproducción de células, meiosis, y espermatogénesis. Una gónada de 50 años habrá visto $40 \%$ más divisiones que una de 40 . Además, con más edad el ADN no autocorrige los errores con la misma eficacia.

En 2001, Malaspina et al. encontraron, en un estudio grande $(N=87.907)$, que los hombres de más de 50 tenían tres veces más posibilidades que hombres de menos de 25 años de engendrar un hijo esquizofrénico, y esta posibilidad se incrementa gradualmente con la edad. Un meta-análisis de 2011 confirmó que el riesgo relativo de esquizofrenia aumentó de 1.84 a 4.62 en la descendencia de padres de más de 35 años.

La EPA ha sido identificada como factor de riesgo para otros trastornos psiquiátricos como el trastorno bipolar, fobias y el trastorno obsesivo-compulsivo, así que aparece como un factor de riesgo no específico, posiblemente relacionado a la mutación genética (Hubert, Szöke, Leboyer, y Schürhoff, 2011). Un estudio de registro sueco de $2014(N=2.615 .081)$ también encontró un incremento del riesgo para trastornos mentales en la descendencia de hombres más mayores (D’Onofrio, Rickert, Frans, Kuja-Halkola, Almqvist, et al.) pero, aunque los riesgos relativos pueden ser 3 o 4 veces más elevados, los riesgos en términos absolutos siguen siendo bajos.

La edad paterna demostró ser relevante para la mortalidad antes de 5 años de la descendencia, en un estudio danés grande de registro de cohorte de 2013 ( $N=$ 1.575.521) (Urhoj, Jespersen, Nissen, Mortensen, y Nybo Andersen, 2013). Los hijos de padres de 40 años o más tenían un riesgo más elevado de morir en la infancia temprana a causa de un riesgo mayor de anomalías congénitas fatales, cánceres y causas externas, como resultado de mutaciones puntuales específicas. Los datos indican que el mismo proceso biológico de selección de células de esperma sanas deteriora con la edad.

Desde el momento en que se produce un gameto, la epigenética empieza a tener su influencia. Los padres más maduros han tenido más tiempo para absorber contaminación, disruptores endocrinos, químicos y metales pesados. Puede que hayan sufrido más estrés, tanto por su ascenso a conseguir y mantener una prosperidad económica o por ser irremediablemente pobre. Todas estas influencias externas sobre el ADN y ARN pueden producir epimutaciones.
Considerando los datos arriba reflejados, el futuro hijo siempre tendrá "células defectuosas" a que enfrentarse, pero más si sus padres son mayores y/o tienen un historial de estilo de vida malsano. Como señalamos antes, el riesgo de defectos se incrementa si se concibe con la ayuda de TRA. En 2012, Davies, Moore, Willson, Van Essen, Priest, et al. determinaron que el 8.3 por cien de los niños nacidos con TRA tenían defectos comparado con el 5.8 por cien de los nacidos sin estas técnicas, un dato ya conocido por otros estudios.

\section{Métodos y técnicas: Posibilidades y probabilidades}

Un breve repaso del estado actual de procedimientos y técnicas relevantes para el tema puede clarificar hasta qué punto los debates acerca de la procreación aplazada son realidades actuales o, por el contrario, aún meras probabilidades futuras. El dato relevante que resulta es que el envejecimiento aparece como el tema más importante en fertilidad para el que existen soluciones posibles aunque parciales, tales como la criopreservación y la donación de gametos.

A partir de la institucionalización del control de natalidad, la sociedad occidental empezó a separar formalmente la procreación y la actividad sexual, aunque la reproducción misma aún se deja en gran medida al azar o a la intervención ad hoc. Sin embargo, como indicamos atrás, desde 2004 los servicios y clínicas de fertilidad están respondiendo al aumento de la demanda por parte de la población en general de la criopreservación de oocitos y esperma, en su modalidad de "congelación social", es decir, sin indicación médica (Gootman, 2012).

\section{Las opciones de la alternativa criobiológica: Esperma, embriones, oocitos y tejido reproductivo}

Cincuenta años de congelar esperma humano (Sherman, 1973) ha producido un abanico de tecnologías de relativamente bajo coste, con resultados que se consideran aceptables (Boitrelle, Albert, Theillac, Ferfouri, Bergere, et al., 2012). Descubrimientos recientes indican que el daño que producían al ADN puede limitarse con el uso de técnicas adecuadas de criopreservación y selección de esperma (Di Santo, Tarozzi, Nadalini, y Borini, 2012). Por tanto, en un futuro no distante, el esperma criopreservado puede que dé mejores resultados que la fertilización con esperma fresco pero de más edad.

La capacidad de congelar, criopreservar, descongelar y realizar embarazos con embriones pre-implantados es una ayuda normal en el tratamiento de fertilidad. Se pre- 
fiere la transferencia electiva de un único embrión, lo cual necesita una mejor calidad de embriones y por lo tanto se han promovido mejores y más selectivos sistemas de cultivo y métodos de criopreservación más adecuados (Human Fertilisation and Embryology Authority, 2010).

Aunque las tasas de éxito de criopreservación, de tanto los oocitos fertilizados como los no fertilizados se han incrementado y son completamente comparables con los resultados con oocitos frescos fertilizados con ICSI (Cobo y Diaz, 2011), aún queda margen de mejora. Los límites legales que se están poniendo a la transferencia múltiple de embriones y el alto coste de los procesos clínicos y de preservación, favorecen la criopreservación de oocitos no fertilizados. La disponibilidad de métodos y técnicas significativamente mejorados implica que, en un futuro no muy distante, también los óvulos jóvenes criopreservados puede que den mejores resultados que la fertilización de óvulos frescos pero de más edad.

Se considera la criopreservación de tejido ovárico con subsecuente auto-trasplantación una forma prometedora de preservar la fertilidad en mujeres antes del tratamiento de una enfermedad maligna, y ya ha producido un número de niños sanos. Se está desarrollando un procedimiento similar para tejido testicular, también del trasplante de células madre dirigido a estos tejidos (Andersen, Kristensen, Greve, y Schmidt, 2012;). Otras soluciones para ciertos problemas de infertilidad se van acercando a la realidad (Woods y Tilly, 2012).

\section{El lado positivo de la criopreservación}

En ciertos casos, la congelación ha llegado a ser equivalente a vida. Personas que sólo hace 30 años hubieran tenido que elegir entre una terapia para salvar la vida o poder tener un hijo, en muchos casos ahora pueden tener ambos. La criopreservación es un instrumento valioso en el tratamiento de un número de problemas médicos con consecuencias directas para la fertilidad y la procreación (Jensen, Morbeck, y Coddington III, 2011). Para un grupo considerablemente más grande, la congelación social se presenta como una opción interesante con que combatir la pérdida causada por edad, estilo de vida e influencias del entorno (Johnson y Tough, 2012). Tan reciente como en 2009 , la criopreservación aún se consideraba una técnica "especulativa" por parte de la American Society for Reproductive Medicine (ASRM) y voces representativas alegaban que "congelando óvulos por razones sociales, las mujeres estarían arriesgándose su futuro" (Ledger, 2009). Sin embargo, en 2013 la ASRM cambió de parecer drásticamente y la misma voz representativa declaró que la criopreserva- ción "deberían considerarlo las mujeres de treinta y pocos años, con o sin pareja, que consideren aplazar el embarazo, y las que consideren extender su familia en algún momento futuro" (Ledger, 2013). Consideramos que no se debe tomar decisión alguna en esta materia sin contemplar las consecuencias a largo plazo del posible daño al futuro niño, tanto por el aplazamiento como por la criopreservación. Conservar la fertilidad debería implicar conservar la calidad.

\section{El lado negativo de la criopreservación}

Como se mencionó antes, los métodos de criopreservación actualmente utilizados han demostrado tener efectos negativos tanto sobre el esperma como los oocitos. Sin embargo, en varios casos se desarrollaron nuevos y mejores métodos que limitan o previenen crio-lesiones, un desarrollo que se prevé que continuará (Isachenko, Isachenko, Petrunkina, y Sanchez, 2012).

Algunos de los efectos de la criopreservación, especialmente el daño a la cromatina/ADN, necesitarán estudios longitudinales de seguimiento para evaluar su impacto sobre la nueva vida. Existen indicaciones que la TRA por sí mismo puede tener algún impacto negativo, y la próxima década deberá clarificar los riesgos específicos de cada técnica y tratamiento específico.

Una aproximación prudente a la posibilidad de utilizar criotécnicas (en realidad, todas las técnicas de laboratorio) por circunstancias sociales (es decir, no médicas y no urgentes) presenta dos aspectos de primera importancia. En primer lugar:”¿Cómo compara el riesgo de un posible daño por aplicar crioprocedimientos a espermatozoides y oocitos, con el deterioro por edad y exposición a un estilo de vida y entorno?" En otras palabras, querremos saber si la calidad de los gametos sufre más con la criopreservación que con el paso del tiempo. La segunda cuestión trata del coste económico del uso de criopreservación para combatir los efectos de envejecer.

\section{Implicaciones (bio)éticas}

Un aspecto ético de primer orden con la aplicación de técnicas de reproducción asistida ha sido, y aún es, la disposición de los embriones creados, razón por la que se ha estado poniendo límites legales a ella. Para algunos la creación (y la destrucción) de embriones cae en un “área moral gris", igual que la contracepción, el aborto o cualquier forma de interferencia con y manipulación de la fertilidad, mientras para otros el deseo de tener un hijo es y debe ser reconocido como un derecho reproductivo prevalente y sumamente privado. La criopreservación para la conservación de la fertilidad en general sólo es 
una entre muchas técnicas utilizadas en la reproducción asistida. Ante la señalada tendencia de utilizarla para fines sociales, primero habría que valorar las posibilidades reales de una aplicación general y social. Un supuesto uso generalizado conllevaría un cambio importante del marco médico, legal y social actual por implicar el reconocimiento de obligaciones implícitas de los futuros padres hacia el "futuro hijo". Significaría un cambio paradigmático en la forma en que la sociedad considere la procreación, por reconocer explícitamente que la procreación no es la mera consecuencia de la actividad sexual o de intervenciones ad hoc en caso de problemas. Este nuevo marco necesita de formas y contenidos socialmente y legalmente configurados, también en países donde la "congelación social" ya ha sido aceptada formalmente, como los Países Bajos (Sheldon, 2010) o Israel (Shkedi-Rafid y Hashiloni-Dolev, 2012). Nuestra sociedad en mayor medida considera al embarazo un asunto privado y sólo adquiere, a través del Estado, un tutelaje secundario una vez el niño ha nacido. Lo que futuros padres puedan o deban hacer respecto a su descendencia, se deja a su discreción. Si una mujer embarazada quiere tomar drogas que son perjudiciales para su bebé, no es castigada. No se le deniega el derecho a ser madre, una y otra vez si quiere, aunque esté claro que con ello está arriesgando la vida o la salud del feto. Lo mismo aplica a los hombres. Esta realidad contradice los requisitos estrictos y eliminatorios que se aplican a candidatos-padres para la adopción en muchos países, donde estilo de vida, abuso de sustancias, peso corporal, salud general, edad y estatus económico cuentan hacia la elegibilidad, mientras los padres naturales no se enfrentan con estos temas.

Hace dos décadas, la Conferencia Internacional sobre Población y Desarrollo de Naciones Unidas (1994) calificaba la salud reproductiva como estar amparada "por derechos humanos" y que las personas están capacitadas para reproducirse y tienen la libertad de decidir si, cuando y cuantas veces hacerlo. Está implícito en esta última condición el derecho de hombres y mujeres a ser informados y a tener acceso a métodos seguros, eficaces, asequibles y aceptables de planificación familiar de su elección, así como a otros métodos de su elección para regular la fertilidad que no sean ilegales, así como el derecho de acceso a servicios de salud apropiados que facilitan a las mujeres una vía segura por el embarazo y el parto y de a las parejas la mejor oportunidad para tener un hijo sano (párrafo 72). (subrayado añadido). Por consiguiente, "la mejor oportunidad" para ser sano no sólo debe entenderse como un derecho de las personas que quieran reproducirse, sino también como un derecho del futuro niño y una obligación para sus progenitores, por lo que Savulescu llama "Procreative beneficence" (Savulescu, 2001) .

La mejora en el estilo de vida, la calidad del entorno y unas actitudes sociales, relevantes para mantener una óptima calidad de gametos tendrá que venir de forma voluntaria, a través de la educación e información. El riesgo de deterioro por envejecimiento puede reducirse a través de la congelación de gametos en una edad temprana aunque no muy temprana, para evitar el proceso de estimulación hormonal y la recolección de óvulos para la criopreservación cuando un embarazo natural es probable. Una fecha óptima para la congelación sería alrededor de los 30 años.

Los continuados desarrollos en TRA hacen entender que, en un futuro previsible, el posible daño, resultado del manejo y la congelación/descongelación de óvulos, esperma o tejido, será menor que el daño probable incurrido en una persona media a consecuencia de vivir $10 \mathrm{o}$ 20 años más antes de intentar la procreación. A medida de que se acerque este momento, todos los interesados en la procreación y en la salud infantil se enfrentan a la pregunta: “¿Deberíamos recomendar la recolección y criopreservación de gametos a una edad temprana y de esta forma crear un sistema de apoyo de calidad?" Voces relevantes argumentan que existe un deber positivo de los clínicos para informar a sus pacientes de que una mujer de 40 tiene más posibilidades de tener un hijo sano si se usan embriones que se crearon antes de mediados de sus 30, en vez de sus óvulos frescos pero más envejecidos (Lockwood, 2011). Para ser más exacto y como dato clínico biológico: en el caso de considerar la congelación social, ésta debe efectuarse antes de que la calidad disminuya considerablemente, así que antes de los 35 años, y a las mujeres de esta edad no se debe hacer creer que, congelando a los 35 , tendrían una probabilidad considerable de un embarazo sano en una fecha posterior (Mertes y Pennings, 2011).

Una implicación importante de la congelación temprana preventiva o defensiva sería que puede haber más oocitos sanos y jóvenes disponibles para donación, eliminando listas de espera y dudas en cuanto a calidad. Sin embargo, conlleva también cuestiones éticas, especialmente en cuanto a los motivos económicos de la donación o del compartir (Mertes, Pennings, Dondorp, y de Wert, 2012).

\section{Las implicaciones económicas de un uso más general de criotécnicas para la preservación de calidad de gametos}

Recientemente, investigadores en los Países Bajos calcularon el coste para el Sistema nacional de Salud de 
darle a la mujer tres ciclos de hiperestimulación ovárica a la edad de 35 para la congelación de oocitos y luego, a la edad de 40, intentar la concepción natural y, si al año no estaba embarazada, usar los oocitos congelados en FIV, en comparación con mujeres de 40 años que, si no logran concebir después de intentarlo durante 1 año, recibir FIV (van Loendersloot, Moolenaar, Mol, Repping, van der Veen, et al., 2011). Encontraron que la primera opción es más rentable que la segunda. Los óvulos más jóvenes daban mejores resultados a menor coste, incluso contando con el coste de la criopreservación durante cinco años. El estudio sólo calculó el coste de dos momentos de FIV diferenciados por edad pero no contemplaba las otras consecuencias de la procreación aplazada, especialmente la influencia negativa del envejecimiento sobre la integridad del ADN de los gametos y, por ende, sobre la vida nueva y, por ende, sobre el sistema de salud a largo plazo. El asunto por decidir no sólo es si el coste de congelar esperma y oocitos se compense con mejores resultados de TRA (que el estudio dice que sí) pero también si el objeto primario de cualquier decisión en este campo debe ser la preservación de calidad (que sería importante para el niño) en vez de sólo la fertilidad. En el caso de que la calidad se considerara objetivo primario, la congelación social podría aplicarse de forma generalizada a un coste progresivamente menor.

\section{Conclusión}

La influencia negativa del mero paso del tiempo sobre el cuerpo humano y su sistema reproductivo conlleva riesgos para la fertilidad y para el futuro niño. Hasta hace poco, la ciencia médica no podía hacer mucho para cambiar este hecho de la vida, pero esto está cambiando rápidamente, en gran parte por la criobiología.

Por razones médicas y bio-éticas, los posibles méritos de estas nuevas realidades técnicas para la procreación necesitan ser contemplados en un contexto más amplio y no solamente por su capacidad para mantener o restaurar la fertilidad en los casos donde enfermedad, accidente o incapacidad ejerzan una influencia negativa.

Las criotecnologías pueden mitigar la presión psicológica que suele existir en engendrar descendencia antes de una edad determinada. Puede resultar difícil una reproducción temprana dadas las exigencias de nuestra sociedad en cuanto a una formación avanzada, carrera profesional, medios económicos adecuados y además y en no menor medida, vivir una igualdad de género real. La "congelación social" se está convirtiendo en una técnica facilitadora de la procreación aplazada, un instrumento deseable y socialmente aceptable conque ampliar la ventana temporal de fertilidad humana.
Sin embargo, una justificación añadida a favor de la criopreservación social se encuentra en las responsabilidades hacia el niño futuro: los futuros padres deberían intentar concebir sobre la base de su mejor calidad reproductiva, y la sociedad puede querer desempeñar un rol más activo ayudándoles a hacerlo.

Aunque los datos disponibles establecen que la criopreservación en la actualidad causa ciertos daños a gametos o tejido reproductivo, los cuales son comparables con los daños causados por el mero paso del tiempo, se necesitan estudios de población a largo plazo para determinar en qué momento la información fehaciente apoyaría a la criopreservación social.

La procreación aplazada no sólo es cuestión de una adaptación médico-técnica a las presiones sociales y económicas incompatibles con una procreación temprana. También conlleva manifestaciones y alteraciones psicológicos relevantes y, en muchos casos, necesitadas de asistencia por el psicólogo clínico. La colaboración interdisciplinar médico-psicológica aportaría el fundamento esencial para su éxito.

\section{Referencias}

Andersen, C.Y., Kristensen, S.G., Greve, T., y Schmidt, K.T., 2012. Cryopreservation of ovarian tissue for fertility preservation in young female oncological patients. Future Oncology 8 , 595-608.

Bay, B., Mortensen, E.L., Hvidtjørn, D., y Kesmodel, U.S. (2013). Fertility treatment and risk of childhood and adolescent mental disorders: register based cohort study. British Medical Journal 5;347, f3978.

Boitrelle, F., Albert, M., Theillac, C., Ferfouri, F., Bergere, M., Vialard, F., ... y Selva, J. (2012). Cryopreservation of human spermatozoa decreases the number of motile normal spermatozoa, induces nuclear vacuolization and chromatin decondensation. Journal of Andrology 33, 1371-1378.

Campagne, D.M. (2006). Should fertilization treatment start with reducing stress? Human Reproduction 21, 1651-1658.

Chachamovich, J., Chachamovich, E., Fleck, M.P., Cordova, F.P., Knauth, D., y Passos, E. (2009). Congruence of quality of life among infertile men and women: findings from a couple-based study. Human Reproduction 24, 2151-2157.

Cobo, A., y Diaz, C. (2011).Clinical application of oocyte vitrification: a systematic review and meta-analysis of randomized controlled trials. Fertility \& Sterility 96, 277-285.

Daly, I., y Bewley, S. (2013). Reproductive ageing and conflicting clocks: King Midas' touch. Reproductive BioMedicine Online 27, 722-732.

Davies, M.J., Moore, V.M., Willson, K.J., Van Essen, P., Priest, K., Scott, H., ... y Chan, A. (2012). Reproductive technologies and the risk of birth defects. New England Journal of Medicine 366, 1803-1813.

Di Santo, M., Tarozzi, N., Nadalini, M., y Borini, A. (2011). Human Sperm Cryopreservation: update on techniques, effect on 
DNA integrity, and implications for ART. Advances in Urology 2012, 854837.

ESHRE Task Force on Ethics and Law (2012). Oocyte cryopreservation for age-related fertility loss. Human Reproduction 27 , 1231-1237.

Fulford, B., Bunting, L., Tsibulsky, I., y Boivin, J. (2013). The role of knowledge and perceived susceptibility in intentions to optimize fertility: findings from the International Fertility Decision-Making Study (IFDMS). Human Reproduction 28, 3253-3262.

Gootman, E. (2012 Mayo 13). "So eager for grandchildren, they're paying the egg-freezing clinic". New York Times.

Heffner, L.J. (2004). Advanced maternal age--how old is too old? New England Journal of Medicine 351, 1927-1929.

HFEA - Human Fertilisation and Embryology Authority (2010). Fertility Facts and Figures 2008. Acceso 20 de Abril, 2015 de http://www.hfea.gov.uk/docs/2010-12-08 Fertility Facts and Figures 2008 Publication PDF.PDF

Hubert, A., Szöke, A., Leboyer, M., y Schürhoff, F. (2011). [Influence of paternal age in schizophrenia].[Article in French] Encephale 37, 199-206.

Isachenko, V., Isachenko, E., Petrunkina, A.M., y Sanchez, R. (2012). Human spermatozoa vitrified in the absence of permeable cryoprotectants: birth of two healthy babies. Reproduction, Fertility and Development 24, 323-326.

Jensen, J.R., Morbeck, D.E., y Coddington III, C.C. (2011). Fertility preservation. Mayo Clinical Proceedings 86, 45-49.

Johansson, M., Adolfsson, A., Berg, M., Francis, J., Hogström, L., Janson, ... y Hellström, A.L. (2010). Gender perspective on quality of life, comparisons between groups 4-5.5 years after unsuccessful or successful IVF treatment. Acta Obstetricia et Gynecologica Scandinavica 89,683-691.

Johnson, J.A., y Tough, S.; Society of Obstetricians and Gynaecologists of Canada (2012). Delayed child-bearing. Journal of Obstetrics and Gynaecology Canada 34, 80-93.

Johnson, D.R., y White, L. (2009). NSFB methodology report. National Study of Fertility Barriers [Computer File]. Population Research Institute [distributor] The Pennsylvania State University; University Park, PA. Acceso Abril 20, 2015. http://sodapop.pop.psu.edu/data-collections/nsfb/dnd

Kimberly, L., Case, A., Cheung, A.P., Sierra, S., AlAsiri, S., Carranza-Mamane, B., ... y Wong, B.C. (2012). Advanced reproductive age and fertility: no. 269, November 2011. International Journal of Gynecology \& Obstetrics 117, 95-102.

Kong, A., Frigge, M.L., Masson, G., Besenbacher, S., Sulem, P., Magnusson, G., ... y Stefansson, K. (2012). Rate of de novo mutations and the importance of father's age to disease risk. Nature 488, 471-475.

Ledger, W. (2009 Feb 1). In: Fertility experts in moral warning over egg freezing. The Observer. Acceso 20 de Abril, 2015 de http://www.theguardian.com/society/2009/feb/01/ egg-freezing-ethics-fertility

Ledger, W. (2013). Annual Congress of the Perinatal Society of Australia and New Zealand, April 2013. Press Release. Acceso 15 Junio, 2014 de http://www.psanzcongress.com.au/pages/media-releases.php

Liu, K., y Case, A. (2011). Advanced reproductive age and fertility. Reproductive Endocrinology and Infertility Committee; Family Physicians Advisory Committee; Maternal-Fetal Medicine Committee; Executive and Council of the Society of
Obstetricians of Canada. Journal of Obstetrics and Gynaecology Canada 33,1165-1175.

Lockwood, G.M. (2011). Social egg freezing: the prospect of reproductive 'immortality' or a dangerous delusion? Reproductive Biomedicine Online 23, 334-340.

Malaspina, D., Harlap, S., Fennig, S., Heiman, D., Nahon, D., Feldman, D., y Susser, E.S. (2001). Advancing paternal age and the risk of schizophrenia. Archives of General Psychiatry 58, 361-367.

Mertes, H., y Pennings, G. (2011). Social egg freezing: for better, not for worse. Reproductive BioMedicine Online 23, 824-829.

Mertes, H., Pennings, G., Dondorp, W., y de Wert, G. (2012). Implications of oocyte cryostorage for the practice of oocyte donation. Human Reproduction 27, 2886-2893.

Nilsen, A.B., Waldenström, U., Rasmussen, S., Hjelmstedt, A., y Schytt, E. (2013). Characteristics of first-time fathers of advanced age: a Norwegian population-based study. BMC Pregnancy and Childbirth 13, 29.

D’Onofrio, B., Rickert, M.E., Frans, E., Kuja-Halkola, R., Almqvist, C., Sjölander, A., ... y Lichtenstein, P. (2014). Paternal age at childbearing and offspring psychiatric and academic morbidity. JAMA Psychiatry 71, 432-438.

Peterson, B.D., Sejbaek, C.S., Pirritano, M., y Schmidt, L. (2013). Are severe depressive symptoms associated with infertility-related distress in individuals and their partners? Human Reproduction 29, 76-82.

Reynolds RM. (2013) Glucocorticoid excess and the developmental origins of disease: Two decades of testing the hypothesis - 2012 Curt Richter Award Winner. Psychoneuroendocrino$\log y 38,1-11$.

Sandín, B., Chorot, P. y Valiente, R.M. (2012). Transdiagnóstico: Nueva frontera en Psicología Clínica. Revista de Psicopatología y Psicología Clínica 17, 181-184.

Savulescu J. (2001). Procreative beneficence: why we should select the best children. Bioethics 15, 413-426.

Schardt, D. (2005). Delayed childbearing: Underestimated psychological implications. International journal of Childbirth Education 20, 34-37.

Sheiner, E., Shoham-Vardi, I., Hershkovitz, R., Katz, M., y Mazor, M. (2001). Infertility treatment is an independent risk factor for cesarean section among nulliparous women aged 40 and above. American Journal of Obstetrics and Gynecology 185, 888-892.

Sheldon, A. (2010). Dutch medical associations support freezing oocytes for non-medical reasons. British Medical Journal $341, \mathrm{c} 4823$.

Sherman, J.K. (1973). Synopsis of the use of frozen human semen since 1964: state of the art of human semen banking. Fertility \& Sterility 24: 397-412.

Shkedi-Rafid, S., y Hashiloni-Dolev, Y. (2011). Egg freezing for non-medical uses: the lack of a relational approach to autonomy in the new Israeli policy and in academic discussion. Journal of Medical Ethics 38, 154-157.

Silva, L.F., Oliveira, J.B., Petersen, C.G., Mauri, A.L., Massaro, F.C., Cavagna, M., ... y Franco, J.G. Jr. (2012). The effects of male age on sperm analysis by motile sperm organelle morphology examination (MSOME). Reproductive Biology and Endocrinology 10, 19.

Singh, N.P., Muller, C.H., y Berger, R.E. (2003). Effects of age on DNA double-strand breaks and apoptosis in human sperm. Fertility \& Sterility 80, 1420-1430. 
Snijders, R.J., Sundberg, K., Holzgreve, W., Henry, G., y Nicolaides, K.H. (1999). Maternal age- and gestation-specific risk for trisomy 21. Ultrasound in Obstetrics and Gynecology 13, $167-170$

Urhoj, S.K., Jespersen, L.N., Nissen, M., Mortensen, L.H., y Nybo Andersen, A.M. (2013). Advanced paternal age and mortality of offspring under 5 years of age: a register-based cohort study. Human Reproduction 29, 343-350. van Loendersloot, L.L., Moolenaar, L.M., Mol, B.W., Repping, S., van der Veen, F., y Goddijn, M. (2011). Expanding reproductive lifespan: a cost-effectiveness study on oocyte freezing. Human Reproduction 26, 3054-3060.

Woods, D.C., y Tilly, J.L. (2012). The next (re)generation of ovarian biology and fertility in women: is current science tomorrow's practice? Fertility \& Sterility 98, 3-10.

Younis, J.S. (2012). Ovarian aging and implications for fertility female health. Minerva Endocrinology 37, 41-57. 